\title{
Martensite Nucleation at Grain Boundaries Containing Intrinsic Grain Boundary Dislocations
}

\author{
Taejin SONG and Bruno Charles De COOMAN* \\ Materials Design Laboratory, Graduate Institute of Ferrous Technology, Pohang University of Science and Technology, Pohang, \\ 790-784 South Korea.
}

(Received on May 6, 2014; accepted on June 16, 2014)

\begin{abstract}
It is shown that grain boundaries containing intrinsic grain boundary dislocations act as preferential nucleation site for the martensitic transformation. The microstructure and crystallography of BCC $\alpha^{\prime}$-martensite formed in a sensitized AISI 304 stainless steel was studied in detail by means of convergent beam Kikuchi pattern analysis. The orientation relationship between martensite and austenite was determined to be Kurdjumov-Sachs type. The presence of intrinsic grain boundary dislocations was observed at a grain boundary $10.83^{\circ}$ from the exact $\Sigma 11 \mathrm{CSL}$ boundary orientation, where a martensite nucleus formed by the faulting and extension of intrinsic grain boundary dislocations. The selection of the crystallographic variant for the martensite nucleus was not related to a reduction in interfacial energy at nucleation, nor was it related to the accommodation of transformation strain by easy slip in the austenite.
\end{abstract}

KEY WORDS: martensite nucleation; grain boundaries; intrinsic dislocations.

\section{Introduction}

Due to the difficulties in the experimental observation on the nucleation stage of martensitic transformation, the exact physical nature of martensite nucleation has not yet been successfully revealed. ${ }^{1)}$ Kinetics models for martensitic transformation $^{2)}$ are therefore largely based on experimental observations of the martensitic transformation kinetics with the assumption that pre-existing frozen-in embryos are thermally activated. Mechanical contributions to the driving force, leading to a stress-assisted transformation or a straininduced transformation nucleation, can usually be taken into account in these models. Whereas the origin of the frozenin embryo is often not specified in models, attempts to identify the nucleation sites continue to be made. The following microstructural features have been proposed as possible nucleation sites for the martensitic transformation: grain boundaries, ${ }^{3)}$ twin boundaries, ${ }^{4)}$ free surfaces ${ }^{5)}$ and inclusion interfaces. ${ }^{67)}$ Taking into account the characteristic features of the martensitic transformation, Olson and Cohen ${ }^{8-10)}$ proposed that the nucleation of martensite occurs by the dissociation of properly spaced arrays of dislocations. In the case of the FCC to HCP transformation, the entire transformation can be achieved by a simple faulting process. For the FCC to $\mathrm{BCC}$ transformation, Bogers and Burgers suggested that the faulting process included the spreading of the core structure of Shockley partial dislocations over a number of successive $\left\{\begin{array}{llll}1 & 1 & 1\end{array}\right\}_{\gamma}$ planes. $\left.{ }^{11}\right)$ The Olson and Cohen model of the martensite embryo is an array of properly spaced dislocations, subjected to a properly oriented long-range stress

* Corresponding author: E-mail: decooman@postech.ac.kr DOI: http://dx.doi.org/10.2355/isijinternational.54.2394 field. The requirements for the potential homogeneous nuclei in the Olson and Cohen model are rarely satisfied in the interior of crystals of real alloys, such as steels. Grain boundaries, incoherent twin boundaries and inclusion particle interfaces are therefore more likely heterogeneous nucleation sites since these regions have a higher likelihood of providing the required dislocation configuration.

In a study on martensitic transformation in the $\mathrm{Fe}-\mathrm{Ni}$ alloy system, Kajiwara ${ }^{12)}$ reported that the Ms temperature of a polycrystalline specimen was much higher than the Ms temperature of a single crystal, implying that the grain boundaries acted as preferential nucleation sites. Kajiwara also observed that not all grain boundaries had the potency to act as nucleation site. Nucleation only occurred at boundaries having a special character, but the nature of these boundaries was not be specified in his report. Recently, the possibility that annealing twin boundaries and high angle random boundaries might act as preferential nucleation sites for martensite was questioned by Tsuzaki et al., ${ }^{13)}$ who measured the Ms temperatures of bi-crystal specimens containing these boundary. They found that the Ms temperature was not lower for the single crystal specimen. The results of both studies underline the fact that although grain boundaries with a specific character may be able to provide nucleation site for martensite, the exact reason why is still not clear.

More recently, Ueda et al. ${ }^{14)}$ observed that the Ms temperature of a Fe-Ni bi-crystal with a $90 \% /<211>$ symmetric tilt grain boundary was higher than for a single crystal specimen and for a bi-crystal with a symmetric twist grain boundary. The authors stated that the formation of equivalent variants on either side of the tilt boundary were able to maintain the compatibility of the transformation strain across the boundary, a type of nucleation they referred to as 
cooperative nucleation $(\mathrm{CN})$. They argued that this resulted in an increase of the Ms temperature. Later, they verified that $\mathrm{CN}$ was not confined to $90^{\circ} /<2 \quad 1 \quad 1>$ symmetric tilt grain boundaries, but could also be observed at $130^{\circ} /<211>$, $150^{\circ} /<211>$ and $180^{\circ} /<211>$ symmetric tilt grain boundaries. ${ }^{15)}$ Even though the nucleation mechanism of $\mathrm{CN}$ successfully explained the favorable nucleation at the boundaries in terms of strain energy reduction, a detailed description of the boundary character as a potential nucleation site from the viewpoint of embryo formation was not provided in their work. In addition, their observation of a higher Ms temperature for a bi-crystal with a $180^{\circ} /<211>$ or $\Sigma 3$ boundary is contrary to the observation of Tsuzaki et al. ${ }^{13)}$ who clearly demonstrated the very low potency of annealing twin boundaries.

Grain boundaries containing Intrinsic Grain Boundary Dislocations (IGBDs) may act as preferential nucleation site for martensite. ${ }^{9)}$ IGBDs are misfit dislocations embedded in special boundaries such as low angle boundaries and Coincident Site Lattice (CSL) boundaries. These dislocations maintain the lattice matching across the grain boundary. ${ }^{16,17)}$ The presence of IGBD in near-CSL boundaries has frequently been observed by Transmission Electron Microscopy (TEM). ${ }^{17-19)}$ In perfectly planar grain boundaries, IGBDs are not subjected to a long-range stress field as the dislocations in regular grain boundary arrays mutually reduce the individual stress fields. ${ }^{20)}$ In most practical cases, however, grain boundaries are curved and they may even have sharp discontinuities. In these curved or discontinuous regions, the mutual reduction of long-range stress field is less perfect, ${ }^{19)}$ and dislocation configurations suitable for the martensitic nucleation could find themselves in suitable conditions. On the basis of the idea that martensite nucleation could preferentially occur at grain boundaries containing IGBDs, the observation of a higher Ms temperature for bi-crystals with a $<211>$ symmetric tilt grain boundary ${ }^{15)}$ may be related to the presence of potential embryos in this special boundary. Table 1 lists the angle/axis notation used in the work of Ueda et $a l .{ }^{15)}$ and equivalent angle/axis representation for the smallest misorientation. With the exception of the $90 \%$ $<211>$ boundary, the $<211>$ symmetric tilt boundaries can be shown to be CSL boundaries in terms of Brandon's criterion, ${ }^{21)}$ implying that $<211>$ tilt boundaries can contain IGBDs. The presence of IGBDs on a $90^{\circ} /<211>$ tilt boundary should also not be excluded since the presence of IGBDs also applies to boundaries of which the deviation from the exact CSL boundary is far from the upper limit of the criterion for the presence of IGBDs. ${ }^{22,23)}$ In the bi-crystal with a $180^{\circ} /<211>$ boundary used in the work of Ueda et al. ${ }^{15)}$ it is not expected that a perfectly coherent interface was achieved by the diffusion bonding technique used to prepare their bi-crystal. It is highly probable that the IGBDs were present at the twin boundary to accommodate the local orientation deviation resulting from the crystal manufacturing process. The conflicting observation for the twin boundaries can therefore be attributed to the presence of IGBDs on the diffusion bonded twin boundary.

In the present study, the microstructure and crystallography of a single martensite nucleus nucleated at a welldefined grain boundary containing IGBDs was investigated by TEM. The results clearly show that the process of faulting and extension of IGBDs was part of the nucleation process of martensite in this specific case.

\section{Experimental}

The investigation of the role of grain boundaries containing IGBDs as potential nucleation sites for the martensitic transformation requires an alloy in which a very small fraction of martensite exists at room temperature, making it possible to evaluate both the structure of the parent austenite grain boundary and the martensite nucleus. It is well known that austenitic AISI 304 stainless steels transform to HCP $\mathcal{E}$ martensite and that the transformation is greatly enhanced by plastic deformation. The formation of BCC $\alpha^{\prime}$-martensite is induced by plastic deformation at the intersections of $\mathcal{\varepsilon}$ bands. ${ }^{24)}$ The phenomenon of strain- or stress-induced martensitic transformation could not be used in the present study because the local driving force for the transformation is strongly affected by the stress state in the transforming region. Instead, the formation of athermal $\alpha^{\prime}$-martensite was observed in sensitized AISI 304 stainless steel of which the Ms temperature raised by the local Cr-depletion resulting from the precipitation of $\mathrm{Cr}$-rich $\mathrm{M}_{23} \mathrm{C}_{6}$ carbides. ${ }^{25)}$ The $\alpha^{\prime}$ martensite formed in sensitized stainless steel nucleates preferentially at austenite-carbide interfaces. The nucleation of martensite at near-CSL boundaries which contain IGBDs is not affected by carbide precipitation because near-CSL boundaries are immune to carbide precipitation. ${ }^{26)}$ Because the carbides have strong tendency to nucleate and grow faster at grain boundaries, plates of $\alpha^{\prime}$ martensite were frequently observed at random grain boundaries. This fact may disturb the clear distinction between martensite nucleated on grain boundary inclusions and martensite formed by the faulting of IGBDs. Therefore, special care was taken to control the carbide precipitation behavior. The $\mathrm{Cr}$ content in the specimen was low enough to induce the formation of athermal $\alpha^{\prime}$-martensitic. The carbides had to be homogeneously distributed in the specimen and be small enough to prevent martensite nucleation at austenite-carbide interfaces.

Cold-rolled AISI 304 stainless steel was used for the present study. The composition of the steel was $0.05 \% \mathrm{C}, 18.3 \%$

Table 1. Angle/axis notation used in the work of Ueda et al. ${ }^{15)}$ and equivalent angle/axis representation for the smallest misorientation.

\begin{tabular}{cllr}
\hline & \multicolumn{1}{c}{ Equivalent angle/axis pair } & \multicolumn{1}{c}{ angle/axis for CSL } & $\Delta \theta_{\mathrm{d}}$ \\
\hline $90^{\circ} /\left[\begin{array}{lll}2 & 1 & 1\end{array}\right]$ & $42.9^{\circ} /[0.17760 .17760 .9679]$ & $43.6^{\circ} /[0.00000 .00001 .0000]$ for $\Sigma 29 \mathrm{~b}$ & $10.7^{\circ}$ \\
$130^{\circ} /\left[\begin{array}{lll}2 & 1 & 1\end{array}\right]$ & $35.9^{\circ} /[-0.5148-0.51480 .6855]$ & $38.2^{\circ} /[-0.5774-0.57740 .5774]$ for $\Sigma 7$ & $5.6^{\circ}$ \\
$150^{\circ} /\left[\begin{array}{lll}2 & 1 & 1\end{array}\right]$ & $46.7^{\circ} /[-0.6684-0.66840 .3265]$ & $46.7^{\circ} /[-0.6667-0.66670 .3333]$ for $\Sigma 29 \mathrm{a}$ & $0.3^{\circ}$ \\
$180^{\circ} /\left[\begin{array}{lll}2 & 1 & 1\end{array}\right]$ & $60.0^{\circ} /[0.5774-0.5774-0.5774]$ & $60.0^{\circ} /[0.5774-0.5774-0.5774]$ for $\Sigma 3$ & $0^{\circ}$ \\
\hline
\end{tabular}


Cr, 8.28\% Ni, 1.07\% Mn, 0.38\% Si, 0.13\% Mo, 0.35\% Cu, balance, Fe. Cold-rolled sheets with a thickness of $1 \mathrm{~mm}$ were annealed at $800^{\circ} \mathrm{C}$ for $2 \mathrm{~min}$ and furnace-cooled to room temperature. Formation of martensite was done by cooling to $-196^{\circ} \mathrm{C}$.

Electron Back Scattered Diffraction (EBSD) analysis was done on Zeiss Ultra 55 Field Emission (FE) SEM equipped with a TSL EBSD system. The EBSD sample surfaces were prepared by electro-polishing in a solution of $10 \% \mathrm{HClO}_{4}+$ $90 \% \mathrm{CH}_{3} \mathrm{COOH}$ at room temperature. A beam step size of $0.05 \mu \mathrm{m}$ was used for the EBSD measurements.

Thin foils for Transmission Electron Microscopy (TEM) were prepared by mechanical grinding to a thickness of $150 \mu \mathrm{m}$ followed by a twin-jet electropolishing at $50 \mathrm{~V}$ in a solution of $5 \% \mathrm{HClO}_{4}+95 \% \mathrm{CH}_{3} \mathrm{COOH}$ at room temperature. TEM specimens were observed in a JEOL 2010F FETEM operated at $200 \mathrm{kV}$.

\section{Results}

\subsection{EBSD Study and Application of Brandon's Crite- rion}

Figures 1(a) and 1(b) present the EBSD Image Quality (IQ) maps for the specimens of AISI 304 stainless steel after annealing at $800^{\circ} \mathrm{C}$ followed by furnace cooling to room temperature and further cooling to $-196^{\circ} \mathrm{C}$, respectively. In the micrographs, the $\mathrm{BCC}$ phase is highlighted in red and the characteristics of the grain boundaries are indicated by differently colored lines: low angle boundaries with misorientation less than $15^{\circ}, \Sigma 3 \mathrm{CSL}$ boundaries (twin boundaries), CSL boundaries with $\Sigma$-values between 5 to $31 \mathrm{~b}$ and high angle random boundaries are indicated in green, yellow, blue and black, respectively. In the present study, the high angle random boundaries were defined as the boundaries which did not meet Brandon's criterion up to $\Sigma 31 \mathrm{~b}$.

The solid arrow in Fig. 1(a) indicates the presence of BCC phase formed during the slow cooling from annealing temperature. This observation is in accord with the previous reports on the formation of $\alpha^{\prime}$-martensite at grain boundaries in sensitized stainless steel. ${ }^{25}$ ) The martensite plates extended to two grain boundaries, one CSL boundary and a high angle random boundary, making it difficult to deduce where the nucleation of the martensite plates occurred. Further cooling to $-196^{\circ} \mathrm{C}$ resulted in the formation of additional martensite plates, as shown in Fig. 1(b). The presence of pre-existing martensite did not stimulate the martensitic transformation, i.e. autocatalytic nucleation was not observed. The additional martensite plates were observed to nucleate independently in isolated regions. The solid arrow in Fig. 1(b) indicates a region where the martensite nucleated on a CSL boundary. The martensite plate was formed at a $\Sigma 9$ boundary and its growth was stopped when it impinged on a twin boundary. This observation, however, does not support the hypothesis that IGBDs may have acted as the preferential nucleation site for the martensitic formation. This is because martensite plates associated twin boundaries and high angle random boundaries, which do not contain IGBDs, can also be observed in the micrograph. Furthermore, many near-CSL boundaries satisfying Brandon's criterion are observed to be free from the martensitic transformation.
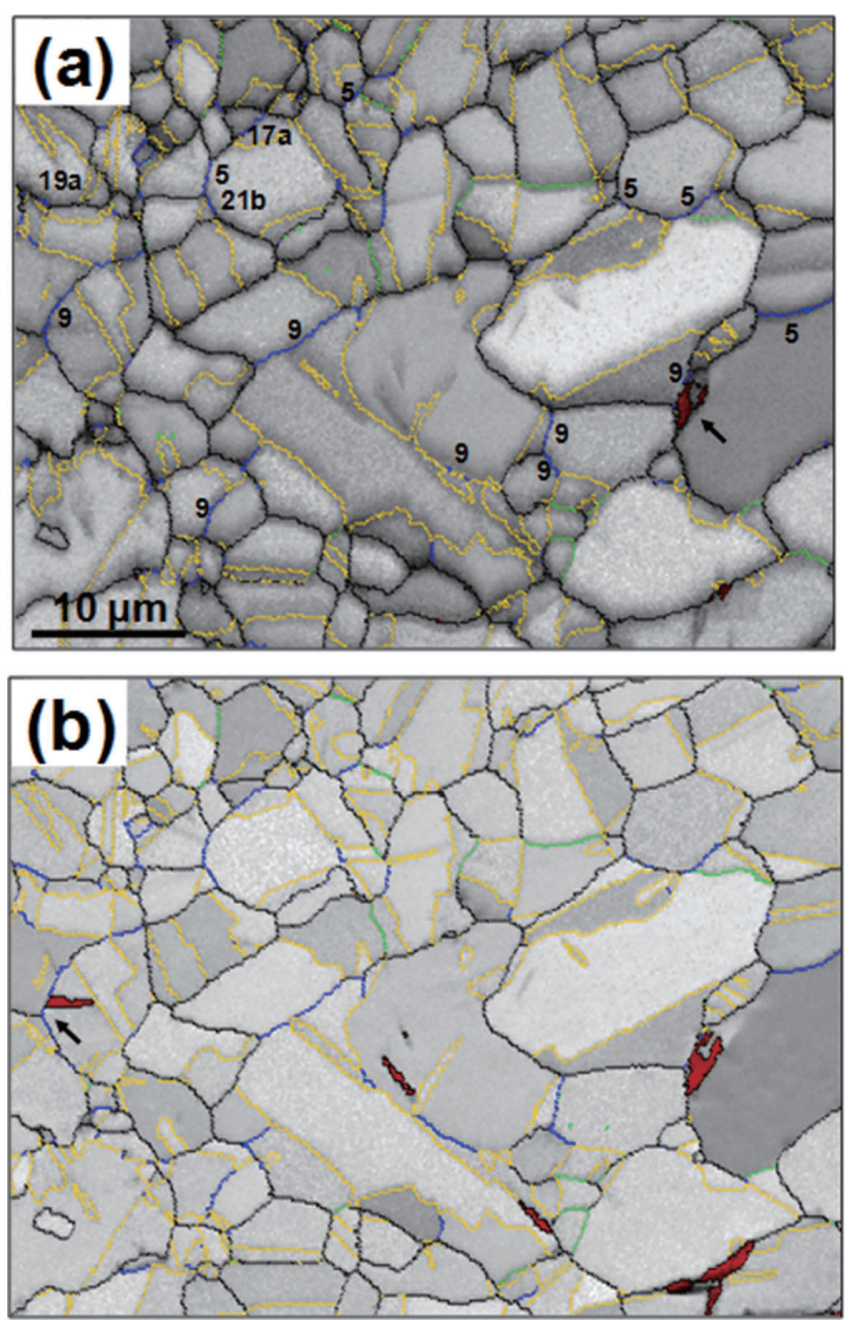

Fig. 1. EBSD IQ maps of AISI 304 stainless steel (a) after annealing at $800^{\circ} \mathrm{C}$ followed by furnace cooling to room temperature and (b) after further cooling to $-196^{\circ} \mathrm{C}$. The $\mathrm{BCC}$ phase is colored red. Green lines indicate low angle boundaries with a misorientation less than $15^{\circ}$. Black lines indicate high angle random boundaries. Yellow lines indicate $\Sigma 3$ CSL boundaries. Blue lines indicate CSL boundaries with $\Sigma$-values from 5 to 31 .

A major difficulty in estimating the potency of grain boundary as a nucleation site for martensite by means of the EBSD technique is that the presence of IGBDs cannot always be predicted by a simple criterion such as Brandon's criterion for the geometrical "specialness" of a boundary. This point is illustrated in the following illustrative example. Figure 2(a) shows a TEM micrograph of a grain boundary in Fe-12Mn-0.6C austenitic steel. The Kikuchi patterns of Figs. 2(b) and 2(c) reveal that these grains were slightly misoriented, with a misorientation across the boundary of $6.3^{\circ}$. According to the Read-Shockley relationship, ${ }^{16)}$ this boundary is expected to conserve its specialness by forming an array of primary IGBDs. However, no evidence for the presence of IGBDs was detected at the boundary. An additional difficulty in applying the EBSD technique is that the presence of small grain boundaries carbides, which could strongly affect the martensitic nucleation, cannot be tested due to the insufficient resolution. 


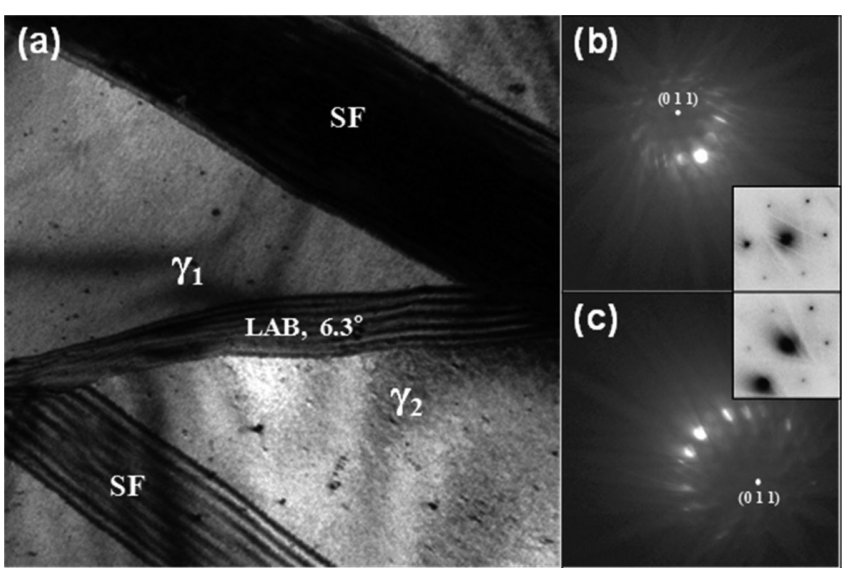

Fig. 2. (a) TEM micrograph for a low angle grain boundary in 12Mn-0.6C austenitic steel, (b) TEM Kikuchi pattern and Selected Area Diffraction (SAD) pattern of the $\gamma_{1}$ grain and (c) TEM Kikuchi pattern and SAD pattern of the $\gamma_{2}$ grain. The misorientation across the boundary was $6.3^{\circ}$. There are no primary IGBDs in the boundary.

\subsection{TEM Analysis of Martensite Nucleation by IGBDs}

Figure 3 shows a view of a TEM specimen of AISI 304 stainless steel annealed at $800^{\circ} \mathrm{C}$ for two minutes, furnace cooled to room temperature, and quenched to $-196^{\circ} \mathrm{C}$. The thermal treatment resulted in the precipitation of $\mathrm{Cr}$-rich carbides during the annealing and slow cooling stages. In Fig. 3 , carbides of uniform size can be seen to be distributed homogeneously in the interior of the austenite grains. A coarse carbide particle precipitated at a grain boundary is indicated by an arrow in the top-middle part of the micrograph. The presence of this coarse carbide at a grain boundary did not stimulate the nucleation of martensite in this area. Instead, a half-lens shaped martensite plate, labeled $\alpha^{\prime}$, was nucleated at the grain boundary between the austenite grains labeled $\gamma_{1}$ and $\gamma_{2}$. The dislocation density is very low except in the immediate vicinity and in the interior of the martensite plate. The possibility that the martensite may have nucleated at a dislocation pile-up ${ }^{27)}$ can therefore be discarded.

Figures 4(a), 4(b), 4(c) and 4(d) present convergent-beam Kikuchi patterns taken in the regions labeled $\gamma_{1}, \gamma_{2}, \gamma_{3}$ and $\alpha$ in Fig. 3, respectively, and corresponding reconstructed stereographic projections showing the corresponding single crystal orientations. The $\left\{\begin{array}{lll}0 & 0 & 1\end{array}\right\},\left\{\begin{array}{lll}0 & 1 & 1\end{array}\right\}$ and $\left\{\begin{array}{lll}1 & 1 & 1\end{array}\right\}$ poles are indicated in the stereographic projections by squares, triangles and hexagons, respectively. The matrices representing the orientation of the crystallites, referenced in the specimen coordinate system, are listed in Table 2. It should be noted that the Kikuchi patterns in Fig. 4 were not taken in the same specimen tilting conditions used for imaging the TEM micrograph in Fig. 3. The tilting condition for Fig. 3 is indicated by the open circles in the stereographs.

Figures 5(a) and 5(b) are the superimposed stereographic projections for the grains $\gamma_{1}$ and $\gamma_{3}$, and for the grains $\alpha^{\prime}$ and $\gamma_{1}$, illustrating the orientation relationship between the grains. In Fig. 5(a), black symbols correspond to $\gamma_{1}$ and gray symbols to $\gamma_{3}$. Boxed areas indicate the crystallographic directions common to $\gamma_{1}$ and $\gamma_{3}$. The stereograph of $\gamma_{1}$ can be brought into coincidence with the stereograph for $\gamma_{3}$ by a rotation of the stereograph for $\gamma_{3}$ through an angle of $60^{\circ}$

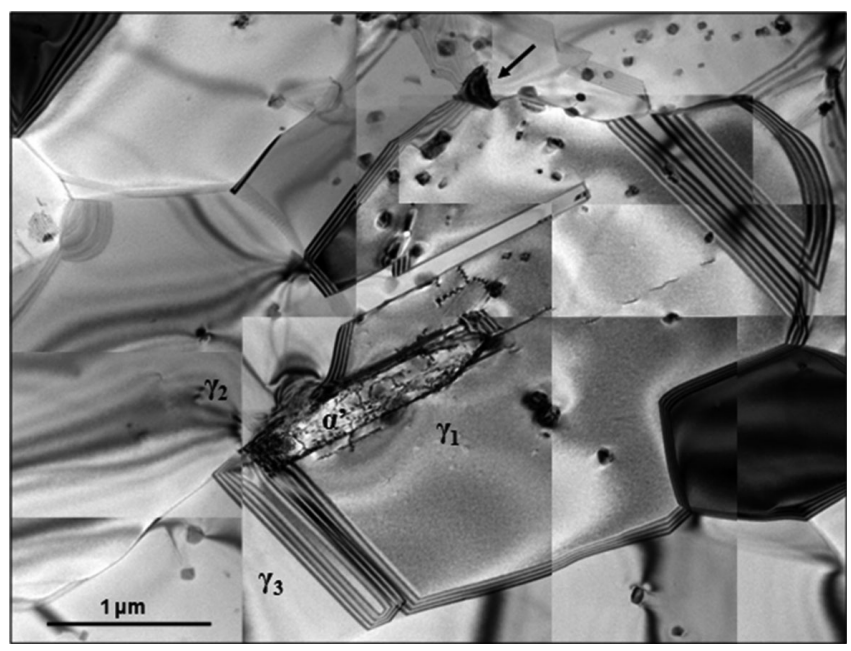

Fig. 3. TEM overview of the grain boundary region where a single martensite lath nucleated in sensitized STS 304 steel. The specimen was annealed at $800^{\circ} \mathrm{C}$ for two minutes, furnace cooled to room temperature and quenched to $-196^{\circ} \mathrm{C}$.

about their common pole $[1-1-1] \gamma_{1}$ and $[1-1-1] \gamma_{3}$. The orientation relationship between the grains $\gamma_{1}$ and $\gamma_{3}$ can therefore be represented by $60 \%[1-1-1]$, the angle/axis notation corresponding to a $\Sigma 3$ twin boundary. Table 3 lists the matrices for the orientation relationship between $\gamma_{1}$ and $\gamma_{3}$, $\alpha^{\prime}$ and $\gamma_{1}, \gamma_{1}$ and $\gamma_{2}$, and $\gamma_{2}$ and $\gamma_{3}$. The experimentally determined orientation matrix deviates by only $0.98^{\circ}$ from an exact twin orientation. Because annealing twin boundaries would normally be expected to be close to the exact CSL, ${ }^{16}$ ) the deviation is considered to be due to the accuracy of the convergent-beam Kikuchi pattern analysis used in the present study. Figure 5(b) shows stereographic projection for the grains $\gamma_{1}$ and $\alpha$, in which black symbols and gray symbols correspond to $\gamma_{1}$ and $\alpha^{\prime}$, respectively. The $\left(\begin{array}{lll}1 & 0 & -1\end{array}\right) \gamma_{1}$ plane normal is perpendicular to $\left(\begin{array}{lll}1 & 1 & -1\end{array}\right) \alpha^{\prime}$, and the $\left(\begin{array}{lll}-1 & -1 & -1\end{array}\right) \gamma_{1}$ plane normal is perpendicular to $(0-1-1) \alpha$. The orientation relationship between the grains $\gamma_{1}$ and $\alpha^{\prime}$ was found to be:

$(-1-1-1) \gamma_{1} 0.90^{\circ}$ deviated from $(0-1-1) \alpha$

$\left[\begin{array}{lll}1 & 0 & -1\end{array}\right] \gamma_{1} 0.92^{\circ}$ deviated from $\left[\begin{array}{lll}1 & 1 & -1\end{array}\right] \alpha$,

For the FCC to BCC phase transformation, the commonly observed orientation relationships are the Kurdjumov-Sachs (K-S) OR, the Nishiyama-Wasserman (N-W) OR and the ORs between $\mathrm{K}-\mathrm{S}$ and $\mathrm{N}-\mathrm{W} .{ }^{28)}$ In the K-S and N-W ORs, the (lll 111 ) $\gamma$ plane is parallel to $\left(\begin{array}{lll}0 & 1 & 1\end{array}\right) \alpha$. The deviation between close packed directions, i.e. [ $\left[\begin{array}{lll}1 & 0 & -1\end{array}\right] \gamma$ and $\left[\begin{array}{lll}1 & 1 & -1\end{array}\right] \alpha^{\prime}$, is $0^{\circ}$ for the K-S OR and $5.26^{\circ}$ for the N-W OR. The deviations reported for intermediate ORs are $2.5^{\circ}$ for the GreningerTroiano (GT) OR, ${ }^{29)} 3.9^{\circ}$ for the Sandvik and Wayman $\mathrm{OR}^{28)}$ and $1.5^{\circ}$ to $3.0^{\circ}$ for the Kelly OR. ${ }^{30)}$ The OR between $\gamma_{1}$ and $\alpha^{\prime}$ observed in the present study confirms the $\gamma-\alpha^{\prime} \mathrm{K}$ $\mathrm{S}$ OR for the martensitic transformation in AISI 304. The deviation between the experimentally determined orientation matrix and matrix for the exact K-S OR is $0.97^{\circ}$ (Table 3 ). This value is comparable to the accuracy of the orientation measurement in the present study.

\subsection{Martensite and Austenite Defect Structure}

Figures 6(a) through 6(f) show the defect structure on the boundary between the grains $\gamma_{1}$ and $\gamma_{2}$. Figures $6(\mathrm{a})$ and $6(\mathrm{~d})$ 

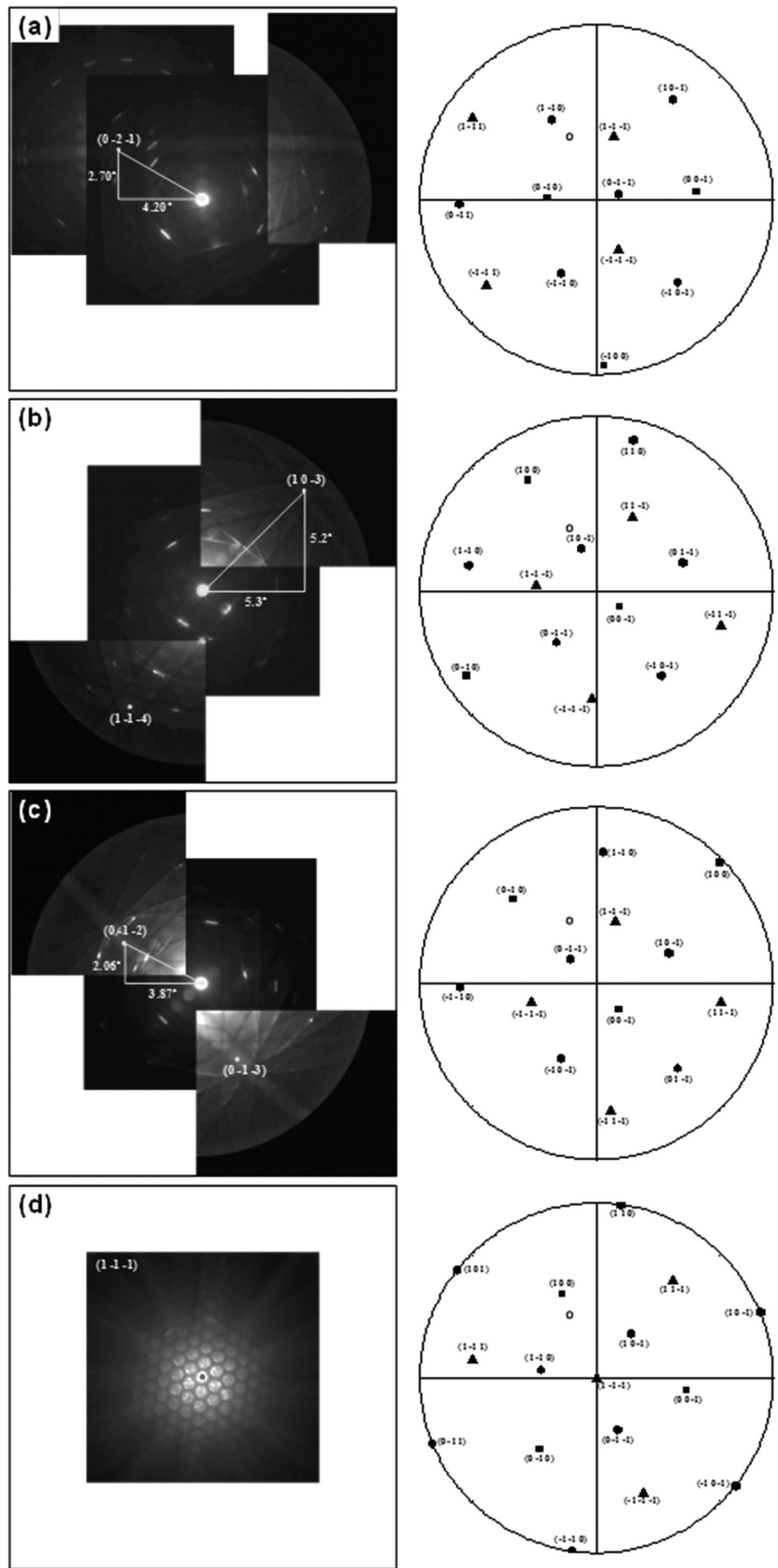

Fig. 4. Kikuchi patterns and stereographic projections representing the orientation of the crystals in Fig. 3. (a) $\gamma_{1}$, (b) $\gamma_{2}$, (c) $\gamma_{3}$ and (d) $\alpha^{\prime}$. Note that the Kikuchi patterns do not correspond to the diffraction condition used for imaging. The orientation for the TEM foil corresponding to BF imaging conditions in Fig. 3 is indicated by the open circles in the stereographic projections.

are TEM BF images taken using the g-vectors $\left(\begin{array}{lll}-1 & 1 & -1)_{\gamma 2} \text { and }\end{array}\right.$ $\left(\begin{array}{lll}0 & -2 & 0\end{array}\right)_{\gamma_{2}}$, respectively. Figures $6(\mathrm{~b})$ and $6(\mathrm{c})$ and $6(\mathrm{e})$ and $6(\mathrm{f})$ are the corresponding DF images. The misorientation matrix for the grains $\gamma_{1}$ and $\gamma_{2}$ is given in Table 3. The boundary between $\gamma_{1}$ and $\gamma_{2}$ deviated by $10.83^{\circ}$ from the exact $\Sigma 11$ CSL boundary. Brandon's criterion states that the 
Table 2. Matrixes representing the orientation of the crystallites in Fig. 3.

\begin{tabular}{|c|c|c|c|c|c|c|c|}
\hline \multirow{3}{*}{$\left(\gamma_{1} J S\right)=$} & $(-0.9975$ & -0.0488 & $-0.0507)$ & \multirow{3}{*}{$\left(\gamma_{2} J S\right)=$} & $(-0.8182$ & -0.4963 & 0.2902 \\
\hline & 0.0188 & 0.5109 & -0.8594 & & -0.5469 & 0.8275 & -0.1268 \\
\hline & 0.0678 & -0.8582 & -0.5087 & & -0.1772 & -0.2624 & -0.9485 \\
\hline \multirow{3}{*}{$\left(\gamma_{3} J S\right)=$} & $(-0.6960$ & -0.7177 & 0.0236 & \multirow{3}{*}{$\left(\alpha^{\prime} \mathrm{J} \mathrm{S}\right)=$} & $(-0.7570$ & -0.3059 & 0.5774 \\
\hline & 0.6575 & 0.6501 & -0.3809 & & 0.6434 & 0.5027 & -0.5774 \\
\hline & -0.2887 & -0.2496 & -0.9243 & & 0.1136 & -0.8086 & -0.5774 \\
\hline
\end{tabular}

(a)

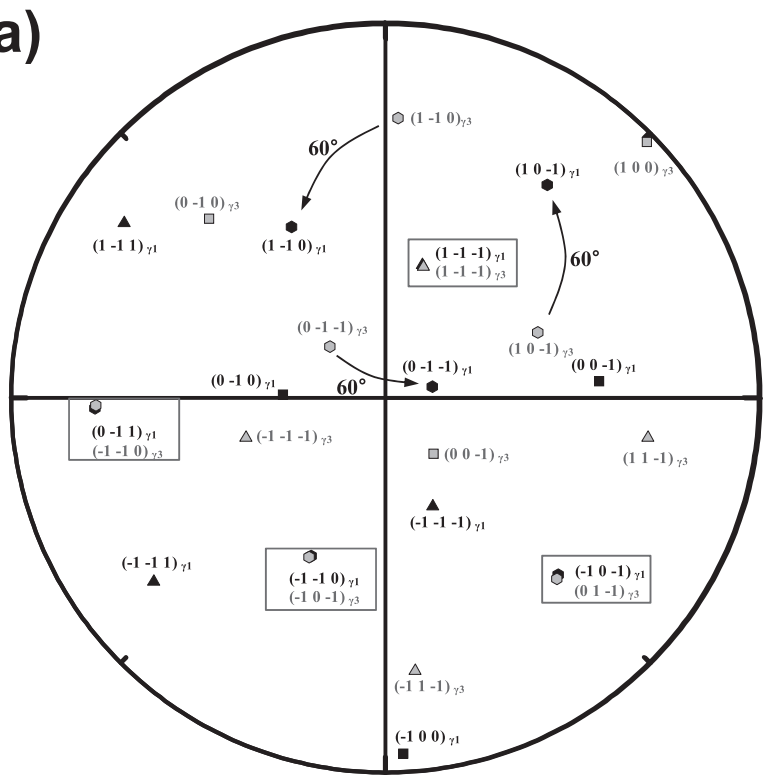

(b)

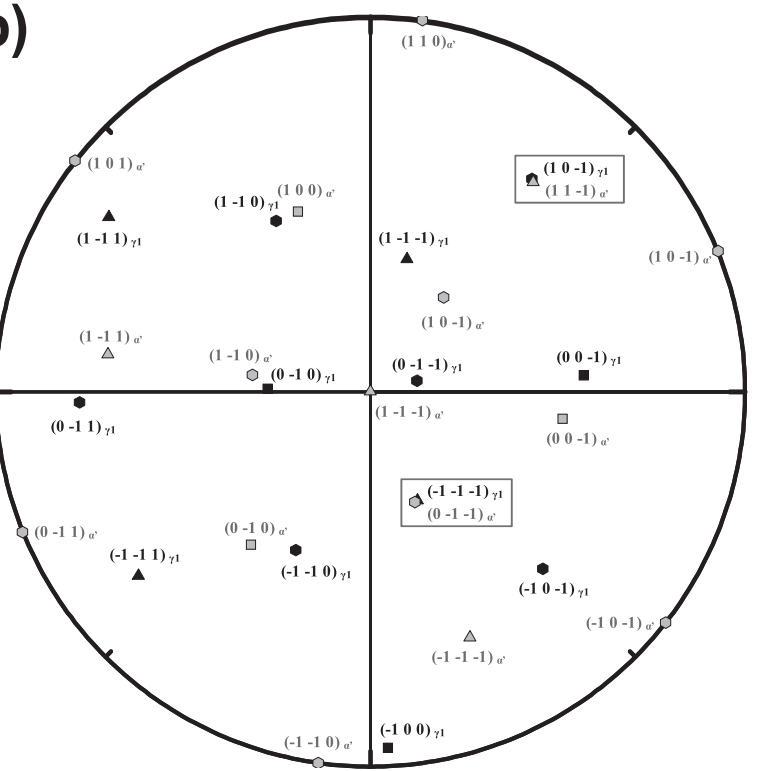

Fig. 5. Stereographic projections showing the orientation relationship between (a) $\gamma_{1}$ and $\gamma_{3}$ and (b) $\gamma_{1}$ and $\alpha^{\prime}$.

special character of a boundary is maintained by adoption of a grain boundary dislocation array up to a maximum angle $\Delta \theta_{\mathrm{d}}$. This angle is $4.52^{\circ}$ for a $\Sigma 11$ boundary. On the basis of this criterion it is expected that IGBDs are not present at the boundary between the grains $\gamma_{1}$ and $\gamma_{2}$. Secondary IGBDs, indicated by solid white arrows, can clearly be seen in Fig. 6(b). Solid black arrows in Fig. 6(b) indicate the presence of primary IGBD at the low angle grain boundary between $\gamma_{2}$ and $\gamma_{3}$. The dashed white arrow indicates the
Table 3. Matrixes representing the orientation relationship between the crystallites in Fig. 3.

\begin{tabular}{|c|c|c|c|c|c|c|}
\hline \multicolumn{7}{|c|}{ Deviation } \\
\hline & $\left(\gamma_{1} \mathrm{~J} \gamma_{3}\right)$ & & \multicolumn{3}{|c|}{$\operatorname{Twin}(\Sigma 3) 60^{\circ} /\left[\begin{array}{lll}-1 & 1 & 1\end{array}\right]$} & \multirow{4}{*}{$0.98^{\circ}$} \\
\hline 0.65805 & -0.66825 & 0.34702 & 0.66667 & -0.66667 & 0.33333 & \\
\hline 0.33339 & 0.67180 & 0.66146 & 0.33333 & 0.66667 & 0.66667 & \\
\hline-0.67515 & -0.31958 & 0.66487 & -0.66667 & -0.33333 & 0.66667 & \\
\hline \multicolumn{3}{|c|}{$\left(\alpha^{\prime} \mathrm{J} \gamma_{1}\right)$} & \multicolumn{3}{|c|}{ K-S (V1) } & \multirow{4}{*}{$0.97^{\circ}$} \\
\hline 0.74078 & -0.66666 & -0.08256 & $(0.74158$ & -0.66667 & -0.07491 & \\
\hline 0.64661 & 0.74095 & -0.18135 & 0.64983 & 0.74158 & -0.16667 & \\
\hline \multirow[t]{2}{*}{0.18207} & 0.08096 & 0.97995 & 0.16667 & 0.07491 & 0.98316 & \\
\hline & $\left(\gamma_{1} \mathrm{~J} \gamma_{2}\right)$ & & \multicolumn{3}{|c|}{$\Sigma 11 \mathrm{CSL} 50.5^{\circ} /\left[\begin{array}{lll}1 & 0 & 1\end{array}\right]$} & \multirow{4}{*}{$10.83^{\circ}$} \\
\hline 0.82565 & 0.51167 & 0.23769 & $(0.81804$ & 0.54562 & 0.18196 & \\
\hline-0.51830 & 0.52148 & 0.67781 & -0.54562 & 0.63608 & 0.54562 & \\
\hline \multirow[t]{2}{*}{0.22286} & -0.68283 & 0.69575 & 0.18196 & -0.54562 & 0.81804 & \\
\hline & $\left(\gamma_{2} \mathrm{~J} \gamma_{3}\right)$ & & & $\Sigma 1$ & & \multirow{4}{*}{$14.52^{\circ}$} \\
\hline 0.97157 & 0.22663 & 0.06850 & $(1.00000$ & 0.00000 & 0.00000 & \\
\hline-0.22005 & 0.97115 & -0.09186 & 0.00000 & 1.00000 & 0.00000 & \\
\hline-0.08734 & 0.07417 & 0.99341 & 0.00000 & 0.00000 & 1.00000 & \\
\hline
\end{tabular}

absence of IGBDs at the random high angle grain boundary between the $\gamma_{1}$-twin and $\gamma_{2}$. The presence IGBDs was also tested in different diffraction conditions as shown in Figs. 6(d) through 6(f).

In-situ deformation experiment on bi-crystals containing a network of GBDs showed that GBDs are able to glide out of a grain boundary under the influence of an applied stress. ${ }^{17)}$ It is therefore highly probable that the first shear in the martensite nucleation involving the displacement of $\mathrm{a}_{\gamma} /$ $18<112>$ on $\left\{\begin{array}{lll}1 & 1 & 1\end{array}\right\}$ plane in the Bogers and Burgers' model can be achieved by a $a_{\gamma} / 2<110>$ or $\mathrm{a}_{\gamma} / 6<112>$ IGBD first gliding out of the grain boundary and then dissociating and spreading into $\mathrm{a}_{\gamma} / 18<112>$ transformation dislocations. The Burgers vectors of GBDs depend on the periodic structure of the grain boundary. In some cases, the GBDs may decompose into GBDs with smaller Burgers vectors belonging to Displacement Shift Complete (DSC) lattice. ${ }^{17}$ ) A grain boundary can therefore consist of various combinations of dislocation networks. As lattice dislocation Burgers vectors are always possible GBD Burgers vectors, it is likely that only IGBDs with a perfect $\mathrm{a}_{y} / 2<110>$ lattice Burgers vector or $\mathrm{a}_{y} / 6<112>$ Shockley-type Burgers vector are capable of nucleating martensite because the glide of other types of dislocations having DCS Burgers vector may not produce atomic configurations appropriate for the $\mathrm{BCC}$ structure. This may explain the EBSD results of Fig. 1 which illustrate 

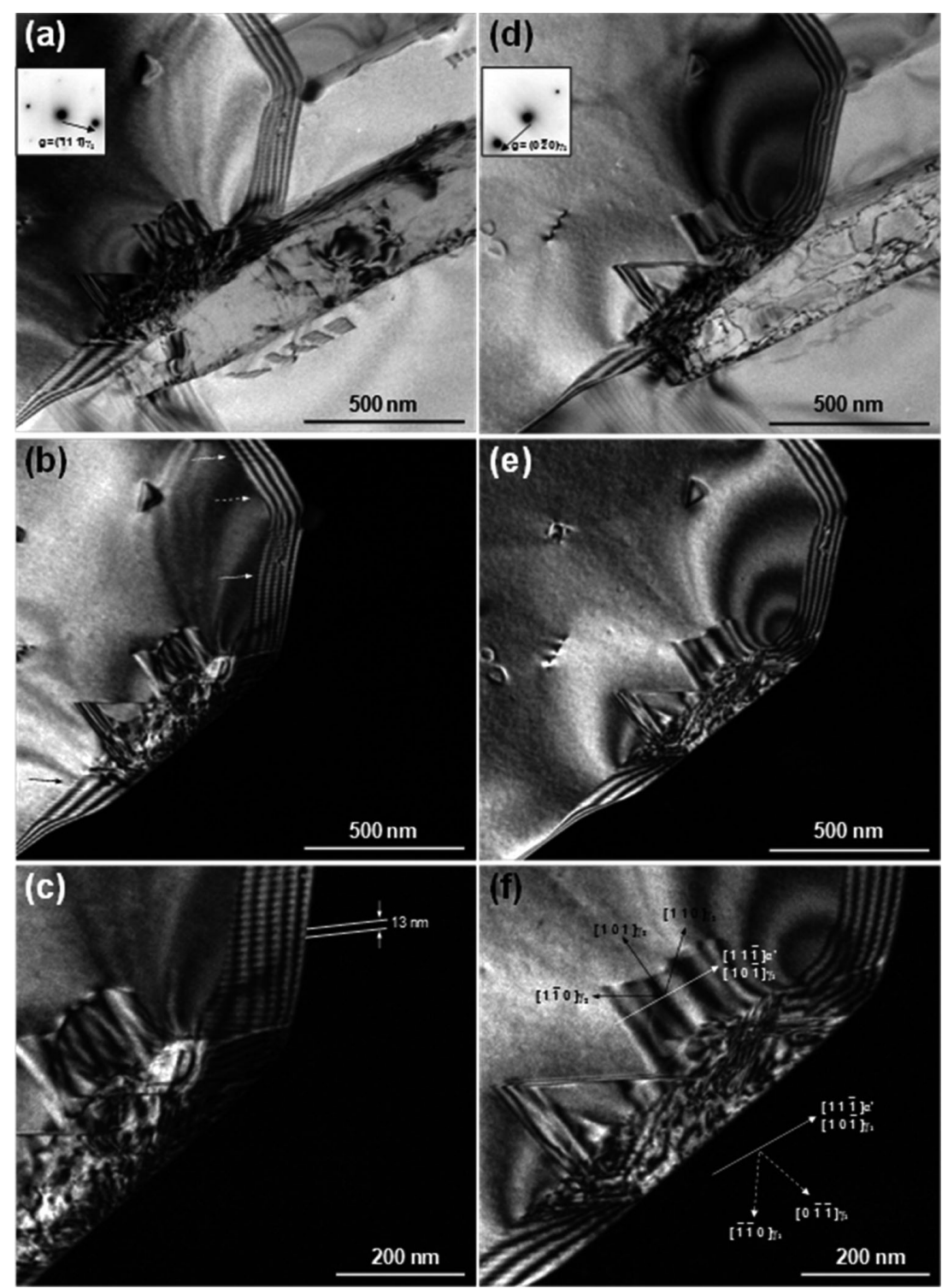

Fig. 6. (a) TEM BF image of the section of the grain boundary between the grains $\gamma_{1}$ and $\gamma_{2}$ where the martensite lath was nucleated, with $\mathrm{g}=\left(\begin{array}{lll}-1 & 1 & -1\end{array}\right)_{\gamma 2}$. (b) DF image of (a). (c) Magnified view of (b). (d) BF image for same area as in (a), with $g=\left(\begin{array}{lll}0 & -2 & 0\end{array}\right)_{\gamma 2}$. (b) DF image of (d) and magnified view of (f). The solid white arrows in (b) indicate the presence of secondary IGBDs in the near- $\Sigma 11 \mathrm{CSL}$ grain boundary between the grains $\gamma_{1}$ and $\gamma_{2}$. The solid black arrow in (b) indicates the presence of primary IGBDs in the low angle boundary between the grains $\gamma_{2}$ and $\gamma_{3}$. The dashed white arrow indicates the absence of IGBDs in the random grain boundary between the $\gamma_{1}$-twin and $\gamma_{2}$.

that only a single type of near-CSL boundary was involved in the formation of martensite plates.

The Burgers vector of the IGBDs in Fig. 6 could not be established but is likely that the IGBDs had a $\mathrm{a}_{y} / 2<110>$ or $\mathrm{a}_{\gamma} / 6<112>$ Burgers vectors since other dislocation arrays were not observed at the grain boundary. In the absence of an alternative dislocation network, this IGBD could easily leave the grain boundary when the sufficient driving force was applied by thermal activation, resulting in a formation of martensite. No dislocations were observed at the phase boundary between $\alpha^{\prime}$ and $\gamma_{2}$. This observation supports the model for the martensitic transformation by a process of faulting and extension of IGBDs into the austenite.

The nucleation and growth of martensite resulted in a formation of crystal defects in the austenite grains $\gamma_{1}$ and $\gamma_{2}$. The crystal defect structure formed in the $\gamma_{2}$ grain is shown in Figs. 6(a) through 6(f). The defects observed in the $\gamma_{2}$ grain are stacking faults which accommodate the transformation strain associated with the martensitic transformation. In Fig. 6(f), the slip directions in the $\gamma_{2}$ grain, $<101>\gamma_{2}$, are indicated by black arrows and the growth direction of martensite, [ $\left[\begin{array}{lll}1 & 1 & -1\end{array}\right] \alpha^{\prime} / /\left[\begin{array}{lll}1 & 0 & -1\end{array}\right] \gamma_{1}$, is indicated by a solid white arrow. The growth direction of the martensite plates makes an angle of approximately $45^{\circ}$ or $90^{\circ}$ with the slip directions in the grain $\gamma_{2}$. If the martensite variant selection was based on a growth direction parallel to $\left[\begin{array}{lll}-1 & -1 & 0\end{array}\right] \gamma_{1}$ or $\left[\begin{array}{lll}0 & -1 & -1\end{array}\right] \gamma_{1}$, the transformation strain would be easily accommodated by intensive slip in the $\gamma_{2}$ grain. The mechanism for variant selection for grain boundary nucleation which favors the martensite variant which maximizes the number of components of transformation strain parallel to the slip direction in the austenite does not appear to operate in the present case. ${ }^{31)}$

The TEM micrographs in Figs. 7 and 8 show the defect 


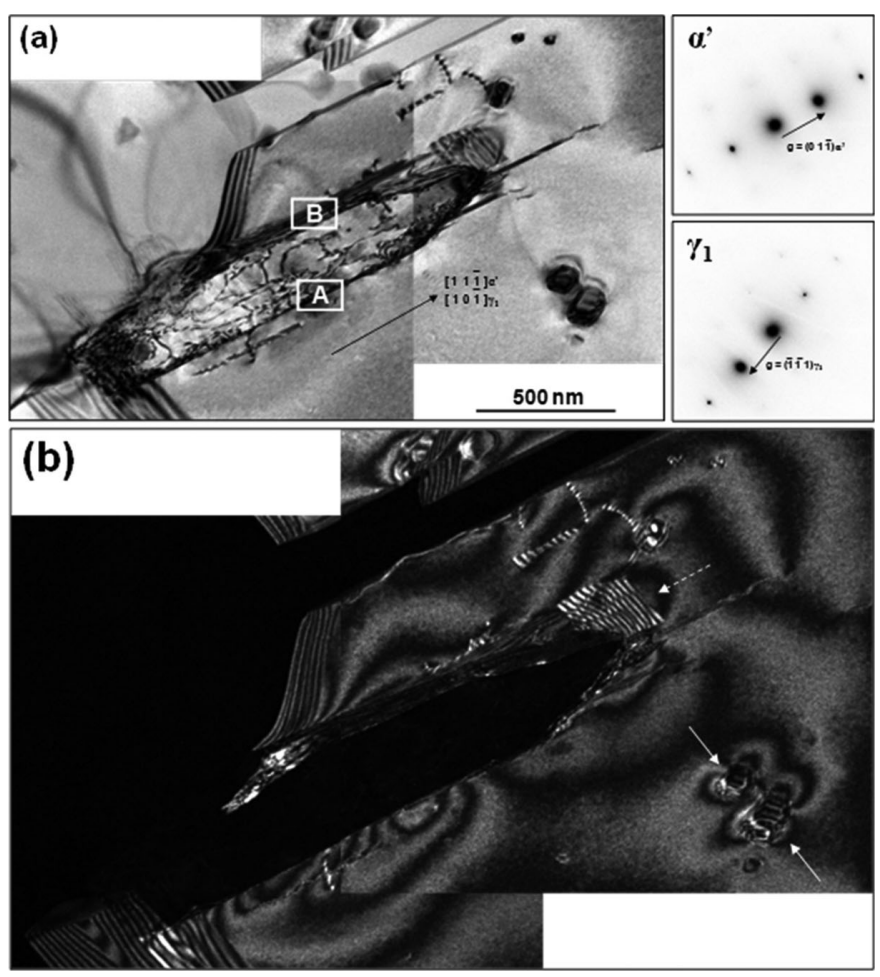

Fig. 7. (a) TEM BF image showing the defect structure in the martensite and austenite. (b) Weak beam DF image of the austenite grain in which the martensite lath grows.

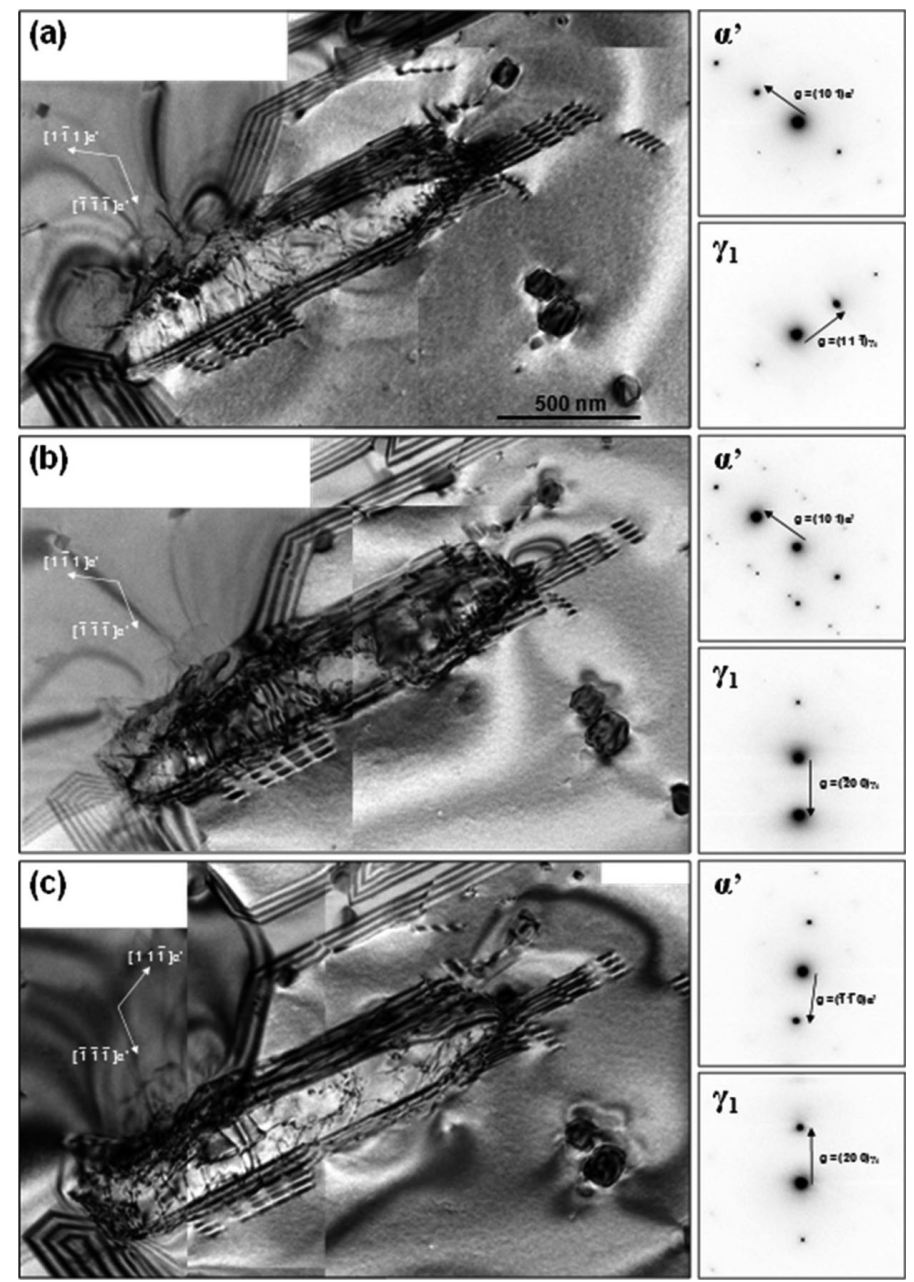

Fig. 8. TEM BF images showing the defect structure in the martensite and in the surrounding austenite grains taken with the primary beam direction close to $\left[\begin{array}{lll}1 & -1 & -1\end{array}\right]_{\alpha^{\prime}}$. (a) g-vectors for $\left(\begin{array}{lll}1 & 0 & 1\end{array}\right)_{\alpha^{\prime}}$ and $\left(\begin{array}{llll}1 & 1 & -1\end{array}\right)_{\gamma 1}$. (b) g-vectors for $\left(\begin{array}{lll}1 & 0 & 1\end{array}\right)_{\alpha}$ and $\left(\begin{array}{lll}-2 & 0 & 0\end{array}\right)_{\gamma 1}$. (c) g-vectors for $\left(\begin{array}{llll}-1 & -1 & 0\end{array}\right)_{\alpha^{\prime}}$ and $\left(\begin{array}{lll}2 & 0 & 0\end{array}\right)_{\gamma 1}$. 
structures in the martensite plate and in the austenite grain $\gamma_{1}$. The BF images of Figs. 7 and 8 show the dislocation structure in the martensite obtained in various two-beam conditions. The beam direction was close to $\left[\begin{array}{lll}1 & 0 & 0\end{array}\right]_{\alpha}$, in Fig. 7. The beam direction was close to $[1-1-1]_{\alpha}$, in Fig. 8. In Fig. 7(a), the Burgers vectors for the dislocations in contrast are indicated by solid arrows. Trace analysis for the dislocation lines indicated that the dislocations in the martensite were mainly of screw character, although they were curved and jogged. This observation supports the findings of previous workers who reported the existence of screw type dislocations in the martensite plates. ${ }^{28,32)}$ Figure $7(\mathrm{~b})$ is a weak-beam DF image for the surrounding austenite. The solid arrows in Fig. 7(b) indicate the presence of the coherency strain field around carbides, revealing that the misfit strain around the carbides was accommodated elastically without generation of dislocations. This result is in accordance with the observation of Furubayashi ${ }^{34)}$ that precipitates smaller than $0.2 \mu \mathrm{m}$ do not generate dislocations which may act as nucleation site for the martensitic transformation. ${ }^{13)}$ Stacking faults generated in the austenite to accommodate the transformation strain associated with the martensitic transformation are indicated by a dashed arrow. One characteristic feature of the martensite plate observed in the present study is that one side of martensite/austenite interface, denoted by A in Fig. 7(a), is observed close to the edge-on condition, whereas the other side, denoted by B, appears to have broad interface in the micrograph. Whereas the plane normal of interface $\mathrm{A}$ is almost perpendicular to $(-1-1-1) \gamma_{1} / /(0-1-1) \alpha^{\prime}$ plane, the interface $\mathrm{B}$ is parallel to the grain boundary between $\gamma_{1}$ and $\gamma_{2}$. This observation is supporting evidence that the martensite plate nucleated by IGBDs leaving the grain boundary to nucleate the martensite.

\subsection{Martensite-Austenite Orientation Relationships}

Figure 9(a) shows a stereographic projection of the K-S variants for grain $\gamma_{1}$. In the figure, gray squares indicate the cube axes of $\gamma_{1}$, the gray circles indicate the $\left\{\begin{array}{lll}0 & 0 & 1\end{array}\right\}$ poles of the $24 \mathrm{~K}-\mathrm{S}$ martensite variants for grain $\gamma_{1}$ and black squares indicate cube axes of the grain $\alpha^{\prime}$. Figure 9(a) confirms that $\alpha^{\prime}$ and $\gamma_{1}$ have K-S OR with $\alpha^{\prime}$ having the orientation of the V1 variant.

Figure 9(b) shows a stereographic projection of the K-S variants for grain $\gamma_{2}$. Gray circles indicate the $\left\{\begin{array}{lll}0 & 0 & 1\end{array}\right\}$ poles of the 24 possible K-S martensite variants for grain $\gamma_{2}$, black squares indicate cube axes of $\alpha$ '. Table 4 lists the possible pairs for K-S variants from $\gamma_{1}$ and from $\gamma_{2}$. The pairs of martensite variants in grains $\gamma_{1}$ and $\gamma_{2}$ with the smallest misorientation are listed in order of ascending misorientation. The martensite variant V13 for grain $\gamma_{1}$ and the martensite variant V15 for grain $\gamma_{2}$ make the smallest misorientation angle of $3.41^{\circ}$. The orientation of the martensite variant V13 for grain $\gamma_{1}$ is indicated by open squares in Fig. 9(b). If the martensite transformation occurred by selection of martensite variant V13 for the grain $\gamma_{1}$, this would also provide a martensite plate orientation with a small deviation from a perfect K-S OR with respect to grain $\gamma_{2}$. This type of variant selection has been observed for the nucleation of diffusional ferrite $^{35)}$ and of upper bainite, ${ }^{31,32)}$ i.e. in conditions where the activation energy for nucleation is reduced by the change
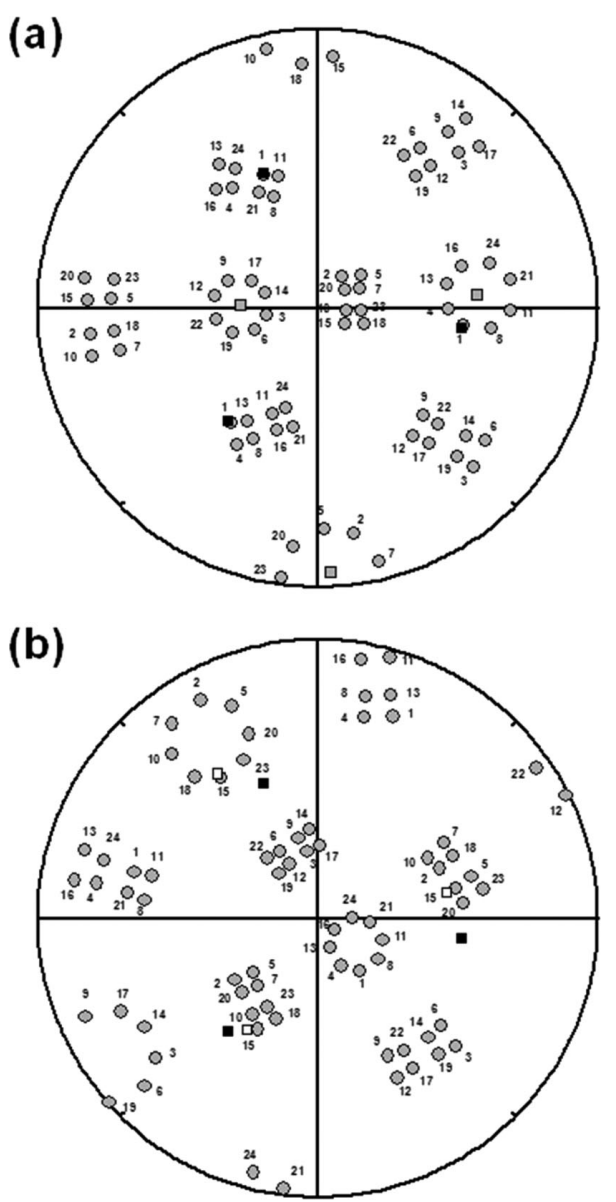

Fig. 9. (a) Stereographic projection of the K-S variants for the $\gamma_{1}$ grain. Gray squares indicate the location of the cube axes for grain $\gamma_{1}$. The gray circles indicate the 24 possible martensite K-S variants for the grain $\gamma_{1}$. The black squares indicate the cube axes for $\alpha$ '. (b) Stereographic projection of the K-S variants for the grain $\gamma_{2}$. Gray circles indicate the 24 possible martensite K-S variants for grain $\gamma_{2}$. The black squares indicate the cube axes of $\alpha$ '. The white squares are for the martensite variant V13 of grain $\gamma_{1}$. This variant has the smallest orientation difference with respect to the martensite variant V15 of grain $\gamma_{2}$.

Table 4. K-S variant pairs for the seven closest misorientations available in the orientation relationship between $\gamma_{1}$ and $\gamma_{2}$. Observed K-S variant in $\gamma_{1}$ was V1.

\begin{tabular}{ccc}
\hline Variant from $\gamma_{1}$ & Variant from $\gamma_{2}$ & misorientation, $\theta$ \\
\hline V13 & V15 & $3.41^{\circ}$ \\
V23 & V21 & $3.68^{\circ}$ \\
V16 & V15 & $7.93^{\circ}$ \\
V15 & V16 & $7.93^{\circ}$ \\
V24 & V23 & $7.93^{\circ}$ \\
V23 & V24 & $7.93^{\circ}$ \\
V16 & V18 & $9.83^{\circ}$ \\
\hline
\end{tabular}

of the grain boundary to a phase boundary. The interfacial energy reduction may not play an important role in the martensite variant selection in the case of martensitic transformations as the observed variant does clearly not provide the largest possible reduction in interfacial energy. The example shown in Fig. 6(f) showed that the martensite variant was also not selected to achieve an easy strain accommodation 
by slip in the austenite. It is clear that in the present case the martensite variant selection resulted solely from the orientation of the IGBDs dislocation line vector relative to the slip planes in the austenite.

\section{Conclusions}

The microstructure and crystallography of a single $\alpha^{\prime}$ martensite lath nucleated at a grain boundary oriented $10.83^{\circ}$ from the exact $\Sigma 11$ CSL boundary in sensitized AISI 304 stainless steel was studied in detail by means of convergent beam Kikuchi pattern analysis. The martensite lath had a K-S OR with respect to the surrounding austenite. Neither interfacial energy reduction nor accommodation of transformation strain by slip in austenite influenced the martensite variant selection. Instead IGBDs were found to act as preferential nucleation site for the martensitic transformation. Only IGBDs with $\mathrm{a}_{\gamma} / 2<110>$ or $\mathrm{a}_{\gamma} / 6<112>$ burgers vectors appeared to nucleate the martensite, by a process of dislocation faulting and extension.

\section{REFERENCES}

1) Z. Nishiyama, M. E. Fine, M. Meshii and C. M. Wayman: Martensitic Transformation, Academic Press, New York, (1978).

2) D. P. Koistinen and R. E. Marburger: Acta Metall., 7 (1959), 59.

3) P. L. Ferraglio and K. Mukherjee: Acta Metall., 22 (1974), 835.

4) S. Dash and N. Brown: Acta Metall., 14 (1966), 595.

5) W. Krauss, S. K. Pabi and H. Gleiter: Acta Metall., 37 (1989), 25.

6) J. Gaggero and D. Hull: Acta Metall., 10 (1962), 995.

7) H. Warlimont: Trans. Metall. Soc. AIME, 224 (1962), 495.

8) G. Olson and M. Cohen: Metall. Mater. Trans. A, 7 (1976), 1897.

9) G. Olson and M. Cohen: Metall. Mater. Trans. A, 7 (1976), 1905.
10) G. Olson and M. Cohen: Metall. Mater. Trans. A, 7 (1976), 1915.

11) A. J. Bogers and W. G. Burgers: Acta Metall., 12 (1964), 255.

12) S. Kajiwara: Metall. Mater. Trans. A, 17 (1986), 1693.

13) K. Tsuzaki, N. Harada and T. Maki: J. Phys. IV France, 5 (1995), C8167.

14) M. Ueda, H. Y. Yasuda and Y. Umakoshi: Acta Mater., 49 (2001), 3421.

15) M. Ueda, H. Y. Yasuda and Y. Umakoshi: Acta Mater., 51 (2003), 1007.

16) V. Randle: The Measurement of Grain Boundary Geometry, Institute of Physics Publishing, Bristol and Philadelphia, (1993).

17) R. W. Balluffi, Y. Komem and T. Schober: Surf. Sci., 31 (1972), 68.

18) W. A. T. Clark and D. A. Smith: Philos. Mag. A, 38 (1978), 367.

19) P. R. Howell, A. R. Jones and B. Ralph: J. Mater. Sci., 10 (1975), 1351.

20) J. P. Hirth and R. W. Balluffi: Acta Metall., 21 (1973), 929.

21) D. G. Brandon: Acta Metall., 14 (1966), 1479.

22) G. Bleris, J. Antonopoulos, T. Karakostas and P. Delavignette: Phys. Stat. Sol. (A), 67 (1981), 249.

23) M. Déchamps, F. Baribier and A. Marrouche: Acta Metall., 35 (1987), 101.

24) P. Mangonon and G. Thomas: Metall. Mater. Trans. B, 1 (1970), 1577.

25) E. P. Butler and M. G. Burke: Acta Metall., 34 (1986), 557.

26) Y. Zhou, K. T. Aust, U. Erb and G. Palumbo: Scr. Mater., 45 (2001), 49

27) M. Suezawa and H. E. Cook: Acta Metall., 28 (1980), 423.

28) B. Sandvik and C. Wayman: Metall. Mater. Trans. A, 14 (1983), 809.

29) S. Morito, X. Huang, T. Furuhara, T. Maki and N. Hansen: Acta Mater., 54 (2006), 5323.

30) P. M. Kelly, A. Jostsons and R. G. Blake: Acta Metall., 38 (1990), 1075.

31) T. Furuhara, H. Kawata, S. Morito, G. Miyamoto and T. Maki: Metall. Mater. Trans. A, 39 (2008), 1003.

32) T. Song and B. C. De Cooman: Metall. Mater. Trans. A, 44 (2013), 1686.

33) K. Wakasa and C. M. Wayman: Acta Metall., 29 (1981), 991.

34) E. Furubayashi: J. Phys. Soc. Jpn., 27 (1969), 130

35) G. Zhang, T. Takeuchi, M. Enomoto and Y. Adachi: Metall. Mater. Trans. A, 42 (2011), 1597. 